\title{
Radiography Students' Learning During Clinical Placements: Developing Professional Knowing in Practice
}

\author{
Lise-Lott Lundvall ${ }^{1,2} \mathbb{D} \cdot$ Nils Dahlström ${ }^{2,3,4} \cdot$ Madeleine Abrandt Dahlgren $^{5}$
}

Received: 6 February 2020 / Accepted: 6 April 2021 / Published online: 6 May 2021

(c) The Author(s) 2021

\begin{abstract}
Radiographers work with image production in medical imaging, a professional field that is undergoing rapid technical development. There is a need to understand how students in radiography education learn within this evolving practice. The aim of this paper is to investigate how radiography students learn professional knowledge in practice during clinical placements. Data collection was through qualitative design using observations and individual interviews. The theoretical framework for the study was a practice-orientated approach. Three themes describing the learning in practice of radiography students emerged as the final result. 1) Attuning to practice: Learning through listening and observing showed how students reconstruct prior knowledge into practical knowing and learn the situated practice. 2) Embodied knowing: Learning through acting in practice illustrated how students reconstructed prior embodied knowledge through their own acting in practice. 3) Dealing with the unexpected: Learning from breakdowns explains how students learn in situations in which unexpected things happen with materiality or relations. On these occasions, relationships with other people were important for developing the students' knowing about the relationship between materiality, actions and people practicing radiography. This study it gives insight into radiography students' learning during clinical placement, which can be useful for planning curricula, as well as clinical learning in radiography education.
\end{abstract}

Keywords Radiographer $\cdot$ Professional knowledge $\cdot$ Learning in practice $\cdot$ Practice theory $\cdot$ Becoming a professional

Lise-Lott Lundvall

Lise-Lott.Lundvall@regionostergotland.se

Extended author information available on the last page of the article 


\section{Introduction}

This study investigates how students training to become radiographers learn at their clinical workplace. In this paper, workplace learning is understood as both individual and collective learning processes in interactions with the culture in practice (Cacciattolo, 2015). Individual learning depends on the learner's own motivation and personal conditions for learning, and collective learning refers to culture and organisational factors that influence learning in workplaces (Billet, 2009; Billet \& Smith, 2006; Billett, 2008a, 2008b). Studies about workplace learning for students in health care are primarily conducted in the largest groups of students in health care - i.e., medical and nursing students. Typically, these studies focus on the organisation and culture in the workplaces where clinical placement takes place (Yardley et al., 2012; Hägg-Martinell et al., 2014; Attenborough et al., 2019). These studies also emphasise relationships and interactions with other professionals, particularly supervisors, as well as personal experiences such as interactions with patients, patient treatment outcomes, feelings about different occasions (Yardley et al., 2012; Hägg-Martinell et al., 2014). To our knowledge, no studies have investigated how materiality such as technology affects workplace learning of students in health care.

This study is about learning during clinical placement of students in training for becoming radiographers. The professional scope of radiographers in Europe, where this study was conducted is described as a dual responsibility for patient care and handling technology during image production and radiotherapy treatment (EFRSa, 2011; EFRSb, 2018). Previous research on radiographers' professional practice illustrate that their professional work compromises caring for patients, handling technology and collaborative work with other professionals in the healthcare sector (Ahonen, 2009; Andersson et al., 2008, 2012a, 2012b). Responsibility for usage of radiation and radiation protection are a part of their professional responsibilities (Ahonen, 2009). Lundvall et al. (2015) illustrate that technology improvement has altered radiographers' professional tasks to being more steered by the technique in use and also taking more responsibility for patient safety for undergo different examinations in medical imaging.

This study was conducted in Sweden where radiographers' professional scope covers medical imaging (Högskoleförordningen 1993:100). The field of medical imaging has changed rapidly in recent decades with the introduction of new technology, which has altered the practice of radiographers and other related professions. The development of techniques includes technological improvements to already-existing modalities of image production. There are also introduction of combinations of different imaging techniques, for example, a combination of Computed Tomography (CT) and Positron Emission Tomography (PET) in one modality of image production (Bentourkia, 2012; Comaniciu et al., 2016). For all modalities in medical imaging, the methods used for image production are constantly developing, which engenders the need to understand how students make sense of this area of professional work as a learning practice. The question of how professional programmes in radiography should be designed and delivered 
in order to best prepare students for entering this professional arena has been debated (Cowling, 2013).

Holmström \& Ahonen (2016) has described in a review article previous research about radiography students' professional learning. These authors show that the focus has mainly been about radiography students' experiences and evaluations of different teaching methods during the theoretical parts of the curriculum. Studies focusing on the learning outcomes of radiography students from their study programmes are rare. Holmström \& Ahonen (2016) argue that research on students' learning outcomes is needed in order to improve radiography education. Furthermore, the review illustrated that the majority of available research studies were quantitative, using various questionnaires that were originally designed for other professions and other contexts. The authors argue that in order to gain in-depth knowledge of how to improve radiography education, there is a need for qualitative studies that focus on radiography students' learning in their professional practice and context (Holmström \& Ahonen, 2016).

\section{Previous Studies}

Former studies on radiography students' learning have focused on how learned theoretical knowledge in form of values and ethics (Mc Inerney \& Lees, 2018) and patient/practitioner interactions was used in practice. Such a focus could be seen as a traditional view of the learning of professional knowledge as the acquisition of theoretical knowledge that is then to be transferred and applied to work within a professional practice (Hager \& Hodkinson, 2011). How students go about reconstructing their theoretical knowledge into practical knowledge is not thematised from this perspective. Some research, for example, Higgins et al. (2013), (2017), has shown how theoretical knowledge about technology is used in experimenting and in discussions among groups of student peers. In these studies (Higgins et al., 2013, 2017), learning professional knowledge appears to be the construction and reconstruction of knowledge, either as individual cognitive processes or as processes whereby professionals learn together and within their milieu (Hager \& Hodkinson, 2011). However, in these studies, the findings indicating that the students had difficulties in demonstrating their knowledge in practice together with professionals could not be explained (Higgins et al., 2013, 2017). Being a part of a team in practice was seen as important for learning in practice (Portainer Mifsud et al., 2015; Hyde, 2015). The students' learning was negatively affected if they had to change between different clinical placements and meet different supervisors. The students stated that, under such circumstances, they did not get to know the staff and had no sense of belonging to the practice (Hyde, 2015). The students' experiences demonstrated that they did not learn in practice whether they could only observe how the radiographers worked without engaging in practical aspects on their own (Portainer Mifsud et al. (2015). This can be understood from a participation perspective on professional learning. From this perspective, professional learning takes place in social situations: Firstly, from legitimate peripheral participation as a newcomer and then moving into full 
participation (Hager \& Hodkinson, 2011; Lave \& Wenger, 1991). These studies (Portainer Mifsud et al., 2015; Hyde, 2015) identified relational aspects of learning, although how the learning was enacted amongst and amid technology-enhanced practice was not explained.

The cited research gives an illustration of how radiography students' learning is, as Gherardi $(2000,2009$, 2015) describes it, situated within a practice that comprises a complex world of relations between actions, people and materiality. This paper will investigate students' learning during clinical placements with the perspective of clinical placements as a way of "becoming professional" through participation, intertwined with their forthcoming professional practice.

Therefore, the aim of this paper is to investigate how radiography students learn professional knowledge in practice during clinical placements.

\section{Theoretical Framework of the Study}

Learning to become a professional radiographer incorporates learning about both technology and relational aspects. In professional educations clinical placements are important because it is learning immersed with people (socio) and things (material) of the coming profession. Thus, a practice theory perspective was chosen as a theoretical framework for data collection and analysis because it takes into account both socio- and material aspects (Schatzki, 2012). From this perspective, knowledge about how to act in practice is semantically, physically and relationally connected and learned through participating in practice (Kemmis, 2005, 2009, 2012). Gherardi $(2000,2009,2015)$ theorises professional practice as being socially and culturally formed. Material objects in the practice, for example, equipment, instruments and other artefacts used during work, are a part of each specific practice. In the practice, actions are performed, and language is used for performing the actions within the practice. Practical actions are undertaken in specific orders, which are formed by the habitus of the practitioners. This can be understood as practice-as-work, which develops a logical structure for continuing the practice. Practice-as-language refers to how language is used for both explaining what is going on and for building relationships between the people in the practice. Finally, practice-as-morality is about authority and politics between the people in the practice (Gherardi, 2000). Knowing (knowledge) in practice mean knowing how to act in a complex world of relations between actions, people and materiality (Gherardi, 2000, 2009, 2015). Acting is being able to perform both the practical doings and use the language in a specific practice. Knowing is learned through negotiation about the meaning of different words, actions, situations and material objects. Practical knowing is developed through the senses and is learned through bodily experiences. These actions have to be repeated several times before the knowing is "set in the body" as a sense of how to perform the actions (Gherardi, 2000). Knowing in a practice is also the ability to use the specific language of the practice. This language is learned through participating in the language game and building relationship with others in the practice (Gherardi, 2000). A knowing person does not think about how to perform these actions, for example, how the material objects in the practice are used, because they 
have developed a sense in their bodies about how to perform the required practical doings and sayings in the practice. It may sometimes be the case that something unexpected occurs that breaks down their feeling about the performance of known actions. The person then has to reflect on prior knowledge and what has happened and change their actions in accordance with the new situation (Gherardi, 2000). The practitioners do not merely perform the required tasks in the practice. Their activities also include emotional, normative and aesthetic values derived from the visions about how the practice should be performed (Gherardi, 2015). Learning how to become a professional has been described as incorporating learning theory, practical skills and morality within a specific professional practice (Hager \& Hodkinson, 2011; Gherardi, 2000, 2015). The metaphor "becoming a professional" can be used for understanding both the gaining of theoretical knowledge and the processes of professional learning in practice (Hager \& Hodkinson, 2011). "Becoming a professional" sees professional learning as arising from both participation in a practice and through the individual learner's construction and reconstruction of knowledge. Thus, Hager and Hodkinson (2011) argue that professional learning comprises a relational aspect as well as an individual aspect (Hager \& Hodkinson, 2011). The individual learning part also includes the development of a professional identity and habitus in a practice (Hager \& Hodkinson, 2011).

\section{Method}

\section{Design}

The project was conducted using a qualitative design that employed observations and individual interviews as the means of data collection. A practice theory perspective about professional knowing in practice (Gherardi, 2000, 2009, 2015) was used as a theoretical framework. Firstly, a case study was conducted employing observations and informal interviews to obtain empirical data from a radiological department as an example of a professional practice in which students are clinically placed. Professional knowing in practice, as described by Gherardi (2000, 2009, 2015), was used for formulating the aim and focus of the data collection in this case study. Secondly, an interview study was conducted comprising individual interviews with radiography students. The interview guide was constructed from the data analysis of the case study and guided by the chosen theory of knowing in practice (Gherardi, 2000, 2009 , 2015). Finally, the data from the case study and the interview study were analysed through an iterative process that moved back and forth between the empirical data and theory (Srivastava \& Hopwood, 2009). 


\section{The Case Study}

\section{Sampling and Data Collection}

The case study was conducted in a middle-sized radiological ward in the Southern part of Sweden. Data were collected by the first author (LL) through observations and informal interviews. One student, in the final year of their radiography education in Sweden, participated in the case study alongside radiographers, nurses and other professionals working in the actual radiological department. The student performed conventional radiological examinations. The total time for data collection was five hours distributed across four sessions. The focus of the observations was the student's actions in practice, and the use of language and negotiations about different situations, words and materiality in the practice. In the informal interviews, LL asked about situations that had recently been observed and how the students had learned from these situations. Field-notes were taken directly after each observation session. The field-notes were organised chronologically and included descriptions of the settings, the persons who participated in the observations and a description of each observation. Data from the informal interviews were written up in chronological order together with the observations from the field-notes.

\section{Data Analysis}

The field-notes and informal interviews from the observations were re-read several times to gain a sense of the data. According to Gherardi (2000, 2009, 2015), knowing in practice is learned through negotiations about the meanings of different words, actions, situations and material things. Thus, the occasions when the students were negotiating about the meanings of different words, actions, situations and material things were identified in the data. These findings from the case study guided the construction of the interview guide for the subsequent interview study.

\section{The Interview Study}

\section{Sampling and Data Collection}

Data were collected through individual interviews with students in their final year of radiography education in Sweden. A total of 111 radiography students from five universities in Sweden received an email containing information about the study. 10 students gave their consent to participate: six after receiving the first email and four after receiving a reminder. The 10 interviewees, eight women and two men, were studying at four different universities. Their median age was 30 years, range 22-44 years.

All interviews were conducted via Skype. The total interview time was $5 \mathrm{~h}$ and $8 \mathrm{~min}$, median time $29 \mathrm{~min}$ (range 20-42 $\mathrm{min}$ ). The interviewees chose the time for the interview. Oral information was given before the interview started. In the "small 
talk" directly after the interviews, the interviewees were asked about their experience of the interview. All the interviewees stated that it been a positive experience. They had chosen to participate in the study because they thought that clinical placement was an important part of their education.

The interview guide included four questions, which were open in nature. The questions were (suggestions for probing questions in italics) as follows:

When you were on clinical placement, what did you get to do that you hadn't experienced in the theoretical part of your education?

Can you tell me about a situation where you got to act as a radiographer. What did you do with the patient? Why? What did you do with the equipment? Was it something that was particularly important? Did the referral have any significance on what you did? Why? What did you do with the images? Why?

How do you act when you don't know what to do? In connection with caring for the patient? In connection with settings in the equipment? In connection with image production? In connection with assessing image quality? How did you know that you had done it correctly? Why was it difficult?

What helped you to learn during your clinical placement?

\section{Analysis of Data from the Case Study and the Interview Study}

The analysis of data was influenced by theoretical thematic analysis (Braun \& Clarke, 2006). The data consisted of two different data sets, the case study and the individual interviews. An iterative process for analysing qualitative data was used throughout the analysis process. This involved moving back and forth between the empirical data and theory (Srivastava \& Hopwood, 2009.

Firstly, in accordance to Braun and Clarke (2006) initial coding of data was performed. Initial coding of the dataset from the case study was performed before construction of the interview-guide. Occasions of events when the student negotiated about meaning of different words, actions, situations and material things were identified in data from the case study. The data in the individual interviews were initial coded through identifying learning events in the data relating to the three different dimensions of professional knowing in practice described by Gherardi (2000): practice-as-work, practice-as- language and practice-as-morality, and were sorted into positive and negative learning experiences in each dimension.

Secondly, in the data set of the individual interviews the codes about positive and negative learning events, were gathered together in potential themes using the question: "What is the data telling me about how the students learn professional knowing in practice?". The potential themes were reviewed together with former identified events of negotiation about the meanings of different words, actions, situations and material aspects in the data set from the case study. Three different themes were identified: learning through a) listening and observation, b) acting and c) at breakdown of former knowledge. These themes were analysed by using the theoretical framework of the study (Hager \& Hodkinson, 2011; Gherardi, 2000, 2009, 2015. 
Thirdly, the themes were defined and named by using the theoretical framework of the study (Hager \& Hodkinson, 2011; Gherardi, 2000, 2009, 2015) in order to illustrate how the radiography students learn professional knowledge in practice.

\section{Ethical Considerations}

The study was conducted in accordance with the Helsinki Declaration and was approved by the Regional Ethics Committee in Linköping (Dnr 2017/197-31). Written information about the case study was sent to the head of the radiological department in which the observations were to be conducted, who gave their consent for the study and informed the employees in the department. The student and the main supervisor received both written and oral information before the data collection started. All interviewees in the interview study received both written and oral information before the data collection started.

\section{Findings}

\section{Attuning to Practice: Learning Through Listening and Observing}

The students emphasised the importance of being able to understand the unique material arrangements and practices of each clinical placement. In order to be able to participate in practice, listening and observing were important initial learning strategies in relation to material objects. This meant seeing physical aspects in the clinical context that they had learned about in their theoretical studies, for example, different types of radiological equipment. They also observed how the radiographers used this specific radiological equipment in the actual clinical placement, how the protocols in the radiological equipment were set and the method descriptions for image production at each clinical placement.

Specific words and terminology, particularly in protocols or in in the radiological equipment, were easier to understand when the students listened to how the professional language was used during practical work. These findings highlight the theoretical standpoint that practices are formed and enacted differently depending on the materiality of each specific practice (Gherardi, 2015). During their theoretical studies, the students learned about the materiality that they might encounter in practice. Observing and listening reconstructed their theoretical knowledge into knowing in practice as they saw how the materiality was organised and how it was used within each specific practice.

In the interviews, the students described how they observed the way in which the radiographers performed their practical work on image production. During their theoretical studies they had learned the relevant theories related to radiographers' work, for example, anatomy, radiation protection, patient care, etc. However, the way in which radiographers perform their work in practice, i.e. the work process, was unfamiliar to the students at each new modality of image production. The sequence 
and order of the practical actions performed by the radiographer appeared to be an important focus for the observations, as indicated by the quote below.

I think that you have to do, I always like that if I come on the first day to a place then it's always at the beginning that I want to see how they do it here. I look and see how they do it and then you see, of course, that many people do it the same way and others do it in different ways so then you try to find, okay, but what do you think should work for me. (Interview 9).

If the students did not have the opportunity to see how the practice was performed before they acted, it resulted in them asking many questions about which actions should be performed, and in which order.

Learning through listening also refers to how the radiographers communicated with patients. The students stated that the ways of communicating with patients differed between different clinical placements, and that their own communication needed to be adjusted accordingly. After observing how the radiographers/supervisors established relationship and communicated with patients, the students initially imitated them but after a while started using their own words and expressions. The students described how they interpreted their theoretical knowledge about caring and communication gleaned from theories about nursing practice by using the parts that they found useful in the radiographers' practice. They could usually tell the outcomes of their actions by the reactions of the patients. They listened to what the patients said and noted whether or not the patients looked confident. They also received verbal or written comments about their communication skills from their supervisors on a daily or weekly basis, although these seldom referred directly to specific situations with patients.

Much of the literature is directed towards, thus the literature that is directed towards radiographers, so it's more with the image production with the positioning but the literature that is available otherwise about the caring part is more directed towards nurses in some ways, so it feels as though you learn more about the caring part as a radiographer at the clinic. (Interview 4).

How they establish contact with the patient or so, it has to be rather fast, like in mammography, you have seven minutes for such things. So I think there are still some differences there if you perform an x-ray examination on someone who has a broken wrist or someone who thinks they have breast cancer. Then you have to be a bit more careful about what you say to people sometimes because people could interpret what you say rather harshly. They worry about it more if they think they have breast cancer or if they think they have a broken wrist. So I think it's rather important and I usually do that on my clinical placement if I've not been there before. I want to hear what they say. (Interview 6).

These findings show that the students learned what Gherardi (2000) refers to as practice-as-work, both as practical actions and as the habitus of the practitioners through the strategies of first observing and understanding the logic before acting in practice. The findings also show that learning through listening, 
Practice-as-language, was important in relation to materiality before the students acted on their own. The students translated their theoretical knowledge about the nursing of caring and communication into practice as language relevant to radiographers' practice. The students learned about practice-as-language (Gherardi, 2000) related to patients through their own acting and participation in practice.

\section{Embodied Knowing: Learning Through Acting in Practice}

The students learned some practical skills related to image production from their theoretical studies by simulating image production with each other. By palpating each other's bodies, they had learned about how different anatomical structures feel and where these were located in the body. They explained that they knew this through their senses. These findings illustrate the embodied nature of professional knowing in practice (Gherardi, 2000). In the interviews, this was described as important for being able to perform image production. They had also learned in theoretical terms how to position a patient correctly before image production in training sessions that were conducted prior to their clinical placement. However, no image was exposed during these training sessions, which meant that the students had not seen the results of their practical actions. When they started performing image production in practice with patients, the students realised the difficulties regarding palpating and positioning the body of an unknown person compared to doing such actions with a student they knew, or a friend. From their theoretical studies, they were able to recognise some of the anatomical structures on the images. However, seeing the images during their clinical placement improved their knowledge of how to perform the necessary practical actions with patients before image production. The students learned this by viewing the images in relation to their own practical actions and the bodies they were visualising. Initially, the supervisors scaffolded the students' observations in order to help them discover different anatomical structures and pathology on the images. Viewing the images together with a supervisor was important for learning how to interpret the results of their practical actions.

Us students practise image production on each other. We were a rather large group and then we should try. We have an x-ray tube and a detector. We centre and we learn how the patient should be placed. For example, whether we should take an x-ray of an ankle. We have learned how we can find the joint that we should centre for image production, the different anatomical parts. The names of the parts in Latin were also included. However we should know this. (Interview 10).

The student said that the simulation was performed at university before the clinical placement. The students then practise settings for image production on each other. What restricts the learning in these training sessions is that the students can never see the image in relation to the settings for image production that they had chosen. The feedback from the image during clinical placement facilitates learning.

(Informal interview during the case study, extract from the field-notes) 
Performance of an X-ray of a wrist and hand with question at issue in the referral request is skeletal injury. The supervisor and the student discussed the content of the referral. The student asked: "How much of the hand should be in the images?" The supervisor said: "In the referral it says that it's only on the lateral part of the hand that the patient feels pain so because of that it's enough to examine that part." The student started performing image production herself and the supervisor remained outside the examination room. The student was not satisfied with the projection of the rotated hand. She discussed this with her supervisor when the image came up on the screen on the modality. The supervisor advised that the hand could be less rotated and suggested that the projection should be re-done. The student did so and the new image was discussed. The supervisor thought it was good, then pointed and indicated what had been visualised better on the new image.

(Field-notes from the case study)

The interviews showed that the practical performance of image production is a process in which things, bodily actions and learning are tightly intertwined. The students learned the necessary actions through what they sense in their own bodies as they performed the examinations. These sensations were about how different anatomical structures felt when palpating the patients' bodies with their hands and how it felt when placing an intravenous peripheral catheter, as well as the sense of touching the technical equipment when preparing for image production. Knowledge of these actions became embodied by repeating the work process several times.

You remember it a different way if you repeat an examination 20 times compared to twice. You remember it and you establish a routine for it and later, when you start working you feel more secure about what you're doing. (Interview 5).

I then have to learn through this motor that I have to click here, I have to press here and I have to turn here. (Interview 2)

The interviews and observations indicate that the students' previous practical knowledge was reconstructed by using their senses during repeated actions in practice. Negotiations with other actors in practice are also important for developing knowledge of materiality. Feeling and sensing materiality during actions and relations with others facilitate the development of knowledge in practice.

Kinsella (2015) has suggested that recognising the role of the body in knowledge production in practice goes beyond focusing on the individual practitioner in the clarification of how the performance of a practice is constituted by the relational nature of material arrangements and professional bodies (Kinsella, 2015). Practice as morality, i.e. the ways in which authority and politics are enacted in practice, influence the students' learning. If supervisors allowed the students to act and perform the examinations on their own, with support when needed, this enabled the embodiment of knowing through repeated actions.

To perform the examination totally independently, I believe in more independence for the student to perform the moments compared to only sitting 
and watching or that you have to constantly be with a supervisor or a radiographer during the training program. Maybe this is good at the beginning of semester one and two, but from semesters three and four it's all about follow the syllabus and try to be independent when you perform these moments independently. When you perform the examination independently you learn more and it will become easier to feel that you are soon complete as staff and you can act as well as or better than them. (Interview 8).

Then we work as a team, colleague to colleague, instead of student and supervisor in the examination room as though you had really completed your training. Then I think I've noticed that it's easier to learn and actually dare. (Interview 9).

\section{Dealing with the Unexpected: Learning from Breakdowns}

Gherardi (2000) explains that a knowing person does not have to think about their actions because they are accustomed to how to act in practice. When something unexpected occurs, it breaks down this familiar sense of how to act and the person has to reflect on former knowledge or experiences in order to know how to change their actions in accordance with the new situation.

In the process of image production, the produced images sometimes did not generate the expected results. This might be because the patient's anatomy/pathology was unusual or because of injuries. It might also be due to technical artefacts on the images. If the patient's anatomy differed from the normal expectations or pathology and the findings/injuries resulted in difficulties achieving the correct visualisation, the students had to interpret the images by using their prior theoretical knowledge of anatomy and pathology. By studying the patient's body and the images, the students tried to remain in charge of the activities and, together with their supervisor, adjust the method used for image production to the actual patient's anatomy or medical status. When it was a totally unknown situation for the student, the new and unusual actions were performed by the supervisor and the students returned to listening and observing. The supervisor explained what they were doing and why these specific actions were used in relation to the actual patient. The supervisor's explanations could refer to actions of a technical nature, for example, changing the parameters in the protocols, as well as adjusting the position of the patient before image production. When a similar situation subsequently occurred, the students remembered and tried to perform these actions on their own.

That it's not possible to do, yes, but these projections in the ordinary way or what shall I say, but you must do it, you must turn the patient a little, a little bit more, or you must angle the x-ray tube a bit more or in that way I usually discuss it a lot with the supervisor. (Interview 4).

The radiographer who worked in that examination room on that day was supposed to help me and he maybe gave me some tips that I hadn't thought about, about placing the patient on a chair, asking the patient to perform a different movement that made it possible to visualise the joint. (Interview 10). 
After the image production, the supervisor and the student studied the images together. The lateral projection was a little too lightly exposed and greyish. The supervisor suggested that it should be a slightly reduced image field with increased exposure. The supervisor changed the exposure parameters in the modality; the student asked what she thought about it but did not receive an answer. Then both of them went in and decreased the image size together, and the supervisor slightly changed the positioning of one of the patient's legs. After the exposure of the image, they studied the new image together and compared it with the previous image. The supervisor said: "This image is much better in exposure and it is not that greyish, either. It turned out like this because we reduced the image size in and changed the positioning of the patient's leg and I also changed the exposure parameters.

(Extract from the field-notes)

These findings show that a breakdown of prior knowledge occurred if materiality did not appear as expected from the performed actions. Then the actors had to interpret the content of the unexpected materiality in terms of previous knowledge. The actor(s) then performed other actions and interpreted the "new" materiality. The students learned through breakdowns whether the practice was organised in ways that made it possible to negotiate with other actors about the actions in relation to the involved materiality.

Breakdowns could also happen when learning about practice as language, in relation to communication with patients. Sometimes patients became distressed due to anxiety, pain or grief. When the students did not know how to handle the complexity of such situations, a similar way of resolving the situation was used as with the material arrangements. The students stepped down from being in charge and let the supervisor take charge instead. The students then listened to what was being said and observed how the supervisor acted to reassure the patient. Having a mutual discussion afterwards supported the students' knowledge of how to handle similar situations later.

There were many occasions when the patients were very scared and many of them broke down in front of us when we were supposed to perform the examination. I believe that for many students it can be difficult, particularly during your training, to really know how to handle such situations, particularly if you are in a new clinical placement that you don't know much about. Then there were some occasions when the patient just stood and totally broke down and you felt that you didn't really know how to respond. So then I let the supervisor take over the complete examination and I later discussed with them how they would think and act on occasions when they [the patients] are so distressed. (Interview 4)

These findings show that breakdowns could occur if the situations were too complex for the students to handle on their own. Seeing, listening and negotiating with other actors in practice about the event supported the students' knowledge in practice. 


\section{Discussion}

The aim of this study was to investigate how radiography students learn professional knowledge in practice during clinical placements.

To summarise the main findings of this study, they illustrate how radiography students learn professional knowledge in practice by alternating between listening to and observing the actions of the radiographers in the practice, and their own acting in practice. The students must have opportunities to alternate between observing/listening and acting on their own when learning during clinical placement. This shows that culture, organisation and relationships between the students and other practitioners are important for the learning processes (Yardley et al., 2012; HäggMartinell et al., 2014; Attenborough et al., 2019).

The most important aspects of establishing knowledge in practice in relation to materiality and professional language appears to be the students' own performance of practical actions, using professional language in relation to other practitioners and patients. Observing and listening appear to be important before they act on their own in order to understand the professional relationships to the material arrangements for each specific practice. This can be seen as mimetic learning, which is described as an important form of learning in practice to enable working in a specific place (Billet, 2014).

Learning through their own acting means developing embodied knowledge, i.e. how to use professional language and how to relate to materiality, to patients and to other professionals in practice. The findings of studies by Higgins et al. (2013, 2017) showed that radiography students found previous theoretical knowledge about technology in the curriculum to be useful in practice. However, these studies did not explain how the students reconstruct prior knowledge into knowing in practice. The findings of this study articulates this comes about through both observing the materiality in practice and by the students' own enacting of the materiality in practice. How practice-as-morality is in each workplace are of importance for students' development of embodied knowledge. Their learning processes are influenced by if they are allowed to act by their own or not.

Knowledge of materiality in radiography practice means learning about how the technical materiality of the practice needs to be arranged differently in order to adapt the technical devices on different patients' bodies to achieve the requested visualisation of the images. Knowledge of materiality also involves the relationship between how the parameters in the radiological equipment are set in relation to the visualised body. When learning this, relationship with other practitioners, for example, the supervisors were important for the development of professional knowledge in practice. This shows connection in between materiality in practice and the socio part. These findings also show similarities between previous studies (Yardley et al., 2012; Hägg-Martinell et al., 2014) about the importance of the relationship with other people in practice for development of professional knowledge during clinical placements.

The students also used observation and listening when breakdowns occurred, such as unexpected things happening when they were practicing. On these occasions, they learned new ways of acting through observing and listening to how 
other practitioners performed the necessary actions. These occasions - in this study described as "breakdown of former knowledge" - share similarities with transformative learning (Mezirow, 2009). The findings in our study show occasions of learning both task-orientated and communicative skills. On both types of occasions it resulted in unexpected and unfamiliar results from the students' activities. In order to resolve these dilemmas, other activities had to be chosen instead. In transformative learning this is identified as taking new perspectives through critical reflection on and assessment of how to identify another solution (Mezirow, 2009). In our findings, the students were not able to carry out these processes independently. Instead, they observed to the supervisors' actions, which can be seen as a form of mimetic learning (Billet, 2014). If the student and supervisor had a discussion afterwards, our findings regarded these occasions as being important for the student's learning. Viewing this from the theory of transformative learning, this is learning based on critical reflection on and assessment of new solutions for resolving the problem. In breakdowns during communications with patients, a similar process occurred. The learning processes that took place in the discussion with others in practice afterwards can be seen as essential informal learning processes (Cacciattolo, 2015). It also shows the relational and interactional parts of learning in workplaces.

The findings of our study add to previous research on how the establishment of knowing in practice in radiography is linked to materiality and to the development of embodied and relational knowledge. Studies by Portainer Mifsud et al. (2015) and Hyde (2015) show that relational aspects are important for radiography students' learning in practice but provide only sparse empirical data about how they learn these skills. Our findings show the importance of relationship to other professionals in practice for the establishment of knowing in practice, i.e. practice-as-morality, for the development of professional knowing.

Reviewing these findings from a socio-material perspective, embodied learning is not enough for learning about materiality during clinical placement for becoming a professional radiographer. When materiality becomes too complex, the students need the socio-part of practice in forms of discussion and explanations from others in practice for developing knowing in practice.

This study combined field studies at one site with individual interviews with radiography students at different universities. This design provided the opportunity to develop an interview guide that was found to be relevant and useful for the purposes of the study. Out of 111 invited interviewees, 10 agreed to participate in the study. One reminder was submitted and a second reminder could have resulted in additional participants who would have been of benefit to the amount of data. This smaller amount of data could affect the transferability of the findings. The participants in the interview study stated that they were specifically interested in the clinical aspects of their educational programmes, which also meant that they had rich narratives to share that strengthen the credibility of the study. A field study of a radiological department and spending more time on fieldwork could have been an alternative design for this study and may have enriched the findings.

Practice theory was chosen as theoretical framework for investigating how radiography students learn professional knowledge in practice, in relation to both material and social aspects. Gherardi's (2000) state that knowing in practice is learned through 
negotiation about the meaning of different words, actions, situations and material objects. This guided us in the case study to recognize the students' activities related to learning the different dimensions of professional practice, i.e. practice-as-work, practice-as-language and practice-as-morality (Gherardi, 2000). Another alternative for the case study could have been to not use a theoretical framework in order to be more open-minded about the empirical findings in the case study. However, combining two sets of data collection was beneficial to verifying the findings in the different data sources. Practice theory was useful for identifying actions and negotiations about the meaning of material objects, actions, language and situations. However, in order to illustrate the findings of how former knowledge was used when learning professional knowledge in practice - both embodied and theoretical - the metaphor "Becoming a professional" (Hager \& Hodkinson, 2011) was found useful for illustrating these learning processes in practice. This can be seen as a limitation in using practice theory. The activities in practice became visible but not the students' processes of reconstruction of former knowledge into knowing in practice. This study may add new knowledge to workplace learning about the role of materiality for learning in practice.

\section{Practical Implications}

This study it gives insight into radiography students' learning during clinical placement, which can be useful for planning curricula, as well as clinical learning in radiography education.

\section{Future Studies}

How radiography students encounter and learn about ethical aspect during clinical placements is sparsely investigated. This topic is useful for educational and professional purposes.

The role of materiality for learning processes in workplace learning could be further investigated.

\section{Funding Open access funding provided by Linköping University.}

Open Access This article is licensed under a Creative Commons Attribution 4.0 International License, which permits use, sharing, adaptation, distribution and reproduction in any medium or format, as long as you give appropriate credit to the original author(s) and the source, provide a link to the Creative Commons licence, and indicate if changes were made. The images or other third party material in this article are included in the article's Creative Commons licence, unless indicated otherwise in a credit line to the material. If material is not included in the article's Creative Commons licence and your intended use is not permitted by statutory regulation or exceeds the permitted use, you will need to obtain permission directly from the copyright holder. To view a copy of this licence, visit http://creativecommons.org/licen ses/by/4.0/. 


\section{References}

Ahonen, S.-M. (2009). Radiographer's work in Finland - A conceptual review. Radiography, 1, 61-65. https://doi.org/10.1016/j.ejradi.2009.09.002.

Andersson, B. T., Fridlund, B., Elgán, C., \& Axelsson, Å. B. (2008). Radiographers' areas of professional competence related to good nursing care. Scandinavian Journal of Caring Science, 22(3), 401-409. https://doi.org/10.1111/j.1471-6712.2007.00543.x.

Andersson, B. T., Christensson, L., Fridlund, B., \& Broström, A. (2012a). Development and psychometric evaluation of the radiographers' competence scale. Open Journal of Nursing, 2, 85-96. https:// doi.org/10.4236/ojn.2012.22014.

Andersson, B. T., Christensson, L., Jakobsson, U., Fridlund, B., \& Broström, A. (2012b). Radiographers' self-assessed level and use of competencies: A national survey. Insights Into Imaging, 3, 635-645. https://doi.org/10.1007/s13244-012-0194-8.

Attenborough, J., Abbott, S., Brook, J., \& Knight, R.-A. (2019). Everywhere and nowhere: Work-based learning in healthcare education. Nurse Education in Practice, 36, 132-138. https://doi.org/10. 1016/j.nepr.2019.03.004.

Bentourkia, M. (2012). Understanding by seeing before treating: Present and future of medical imaging. Computerized Medical Imaging and Graphics, 36, 515-518. https://doi.org/10.1016/j.compm edimag.2012.07.003.

Billet, S., \& Smith, R. (2006). Interdependencies at work: Reflection, performance dialogue and reward. Journal of Adult and Continuing Education, 12(2), 156-169. https://doi.org/10.7227/JACE.12.2.3.

Billett, S. (2008a) Learning throughout working life: A relational interdepence between personal and social agency. British Journal of Educational Studies 56(1) 39-58. https://www.jstor.org/stable/ 20479570. Accessed 6 Feb 2021.

Billett, S. (2008b). Learning through work: Exploring instances of relational independencies. International Journal of Education Research, 47(4), 232-240. https://doi.org/10.1016/j.ijer.2008.07.006.

Billet, S. (2009). Conceptualizing learning experiences: Contributions and mediations of the social, personal and brute. Mind, Culture and Activity, 16(1), 32-47. https://doi.org/10.1080/1074903080 2477317.

Billet, S. (2014). Mimetic learning at work: Learning through and across professional working lives. In S. Billet (Ed.), International Handbook of Research in Professional and Practice-based Learning. Springer.

Braun, V., \& Clarke, V. (2006). Using thematic analysis in psychology. Qualitative Research in Psychology, 3(2), 77-101. https://doi.org/10.1191/1478088706qp063oa.

Cacciattolo, K. (2015) Defining workplace learning. European Scientific Journal, 1, 243-250. https:// eujournal.org/index.php/esj/article/view/5559

Comaniciu, D., Engel, K., Georgescu, B., \& Mansi, T. (2016). Shaping the future through innovations: From medical imaging to precise medicine. Medical Image Analysis, 33, 19-26. https://doi.org/10. 1016/j.media.2016.06.016.

Cowling, C. (2013). Global review of radiography. Radiography, 19, 90-91. https://doi.org/10.1016/j. radi.2013.01.001.

European Federation of Radiographer Societies [EFRS] (2011) EFRS Definition of radiographer. https:// firebasestorage.googleapis.com/v0/b/efrs-f097e.appspot.com/o/publications\%2F912add47-11c34063-9b88-8ce5ef90fae6?alt=media Accessed 2021-01-27

European Federation of Radiographer Societies [EFRS] (2018) European Qualifications Framework (EQF) Level 6 Benchmarking Document: Radiographer. Second Edition. https://firebasestorage. googleapis.com/v0/b/efrs-f097e.appspot.com/o/publications\%2Ffe4ac827-bc8d-4430-8aa6-1f9c8 71a03b8?alt=media Accessed 2021-01-27

Gherardi, S. (2000). Practice-based theorizing and knowing in organizations. Organization, 7, 211-223. https://doi.org/10.1177/135050840072001.

Gherardi, S. (2009). Knowing and learning in practice-based studies: An introduction. The Learning Organization, 16(5), 352-359. https://doi.org/10.1108/09696470910974144.

Gherardi, S. (2015). How the turn to practice may contribute to working life studies. Nordic Journal of Working Life Studies, 5(3a), 13-25. https://doi.org/10.19154/njwls.v5i3a.4831.

Hägg-Martinell, A., Hult, H., Henriksson, P., \& Kiessling, A. (2014). Students perceive health care as a valuable learning environment when accepted as a part of the workplace community. Education for Health, 27(1), 15-23. https://doi.org/10.4103/1357-6283.134296. 
Hager, P., \& Hodkinson, P. (2011). Becoming as an appropriate metaphor for understanding professional learning. In L. . Scanlon (Ed.), “Becoming” a Professional: An Interdisciplinary Analysis of Professional Learning. Springer.

Higgins, R., Hogg, P., \& Robinson, L. (2013). Towards a research informed teaching experience within a diagnostic radiography curriculum: The level 4 (year 1) student holistic experience. Radiography, 19, 62-66. https://doi.org/10.1016/j.radi.2012.08.006.

Higgins, R., Hogg, P., \& Robinson, L. (2017). Research informed teaching experience in diagnostic radiography: The perspectives of academic tutors and clinical placement educators. Journal of Medical Imaging and Radiation Sciences, 48, 226-232. https://doi.org/10.1016/j.jmir.2017.06.002.

Holmström, A., \& Ahonen, S.-M. (2016). Radiography students' learning: A literature review. Radiologic Technology, 87(4), 371-379.

Hyde, E. (2015). A critical evaluation of student radiographers' experience of the transition from the classroom to their first clinical placement. Radiography, 21, 242-247. https://doi.org/10.1016/j.radi. 2014.12.005.

Illeris, K. (2011). The Fundamentals of Workplace Learning. Routledge.

Kemmis, S. (2005) Knowing practice: searching for saliences. Pedagogy, Culture and Society, 13(3):391-426.

Kemmis, S. (2009). Understanding professional practice: a synoptic framework. In B. Green (Ed.), Understanding and researching professional practice (pp. 19-37). Sense Publishers.

Kemmis S. (2012). Researching educational praxis: spectator and participant perspectives. British Educational Research Journal, 38(6);885-905. https://doi.org/10.1080/01411926.2011.588316.

Kinsella, E. A. (2015). Embodied knowledge: Toward a corporeal turn in professional practice, research and education. In B. Green \& N. Hopwood (Eds.), The Body in Professional Practice, Learning and Education: Body/Practice. (pp. 245-260). Springer International Publishing.

Lave, J., \& Wenger, E. (1991). Situated Learning: Legitimate Peripheral Participation. Cambridge University Press.

Lundvall, L-L., Abrandt-Dahlgren, M. \& Wirell, S. (2015) How do technical improvements change radiographers' practice - A practice theory perspective. Radiography, 21, 231-235. https://doi.org/10. 1016/j.radi.2014.12.002.

Mc Inerney, J., \& Lees, A. (2018). Values exchange: Using online technology to raise awareness of values and ethics in radiography education. Journal of Medical Radiation Science, 65, 13-21. https://doi. org/10.1002/jmrs.258.

Mezirow, J. (2009). An overview on transformative learning. In K. Illeris (Ed.), Contemporary theories of learning. Learning theorists...in their own words. Routledge.

Portainer Mifsud, C., Castillo, J. \& Portelli, J. L. (2015) Radiography students' clinical placement experiences in MRI: A phenomenological study. Radiography, 21(1), e17-e20. https://doi.org/10.1016/j. radi.2014.06.012.

Schatzki, T. R. (2012). A primer on practices: Theory and research. In J. Higgs, R. Barnett, S. Billett, M. Hutchings, \& M. Trede (Eds.) Practice-based education: education and strategies. Rotterdam: Sense Publisher.

Srivastava, P., \& Hopwood, N. (2009). A practical iterative framework for qualitative data analysis. International Journal of Qualitative Methods, 8(1), 76-84. https://doi.org/10.1177/160940690900800 107.

Yardley, S., Teunissen, P. M., \& Dornan, T. (2012). Experimental learning: AMME Guide No. 6. Medical Teacher, 34, e102-e115. https://doi.org/10.3109/0142159X.2012.650741.

Publisher's Note Springer Nature remains neutral with regard to jurisdictional claims in published maps and institutional affiliations. 


\section{Authors and Affiliations}

\section{Lise-Lott Lundvall ${ }^{1,2}$ (D) Nils Dahlström ${ }^{2,3,4} \cdot$ Madeleine Abrandt Dahlgren $^{5}$}

Nils Dahlström

Nils.Dahlstrom@liu.se

Madeleine Abrandt Dahlgren

Madeleine.abrandt.dahlgren@liu.se

1 Department of Radiology in Linköping, Linköping, Sweden

2 Department of Health, Medicine and Caring Sciences, Linköping University, Linköping, Sweden

3 Unit of Radiological Sciences, Division of Diagnostics and Specialist Medicine, Linköping University, Linköping, Sweden

4 Center for Medical Image Science and Visualization (CMIV), Linköping University, Linköping, Sweden

5 Division of Society and Health, Department of Health, Medicine and Caring Science, Linköping University, Linköping, Sweden 